\title{
Pesquisa científica em Endodontia apresentada na Reunião Anual da Sociedade Brasileira de Pesquisa Odontológica: análise bibliométrica de 2010 a 2018
}

\author{
Marilisa Carneiro Leão Gabardo*; Felipe Andretta Copelli**; Alini Lisboa Tuzzi***; Graciele \\ Trentin***; Jacqueline Lima****; Flávia Sens Fagundes Tomazinho*; Yara Teresinha Correa Silva- \\ Sousa $* * * * *$
}

\author{
* Professora titular, Programa de Pós-Graduação em \\ Odontologia, Universidade Positivo \\ ** Estudante, Programa de Pós-Graduação em Odontologia, \\ Universidade Federal do Paraná \\ *** Estudante, Graduação em Odontologia, Universidade \\ Positivo \\ **** Estudante, Programa de Pós-Graduação em Odontologia, \\ Universidade Positivo \\ ***** Professora titular, Programa de Pós-Graduação em \\ Odontologia, Faculdade de Odontologia de Ribeirão Preto, \\ Universidade de São Paulo
}

Recebido em 07/06/2019. Aprovado em 24/10/2019.

\begin{abstract}
RESUMO
A Reunião anual da Sociedade Brasileira de Pesquisa Odontológica (SBPqO) é um importante evento da produção científica odontológica no país. A avaliação do perfil dos trabalhos apresentados pode identificar características e a evolução da pesquisa em Endodontia. Por meio desta análise bibliométrica retrospectiva buscou-se investigar a produção científica na área de Endodontia nos suplementos das reuniões da SBPqO, de 2010 a 2018. Os suplementos foram acessados on-line, no sítio web da instituição, e os resumos foram analisados de acordo com a região de origem: Norte, Nordeste, Centro-Oeste, Sul e Sudeste; e categorias temáticas: Terapêutica, Anatomia, Biossegurança, Microbiologia, Citologia/Genética, Diagnóstico, Epidemiologia, Materiais, Técnicas, Instrumentos, Traumatismo e Desfecho clínico. Dos 22.310 resumos publicados no período, foram selecionados 2516 da área de Endodontia. No ano de 2010 houve o maior número de resumos publicados $(\mathrm{n}=375)$. Prevaleceram as pesquisas voltadas à análise de materiais $(27,1 \%)$ e de técnicas $(20,6 \%)$. As regiões Sudeste e Sul apresentaram frequências de resumos com valores de 65,5\% e $17,2 \%$, respectivamente, enquanto a menor participação foi da região Norte $(2,5 \%)$. A frequência de pesquisas de Endodontia publicadas nos resumos dos suplementos da SBPqO se manteve equilibrada ao longo do período analisado, com destaque para objetivos focados em materiais e técnicas e maior produtividade das regiões Sudeste e Sul.
\end{abstract}

Descritores: Pesquisa. Odontologia. Endodontia. 


\section{INTRODUÇÃO}

A pesquisa é componente central e indispensável para a melhoria da saúde e redução de iniquidades ${ }^{1}$, em especial nos países em desenvolvimento, a exemplo do Brasil ${ }^{2}$.

$\mathrm{O}$ investimento em pesquisas científicas na área da saúde no mundo é de bilhões de dólares, com detenção das maiores parcelas para os países desenvolvidos ${ }^{3,4}$. Estados Unidos, Reino Unido e Japão figuram no cenário mundial como os países de ponta na produção científica e tecnológica 5 . Entidades como o Global Forum for Health Research defendem maiores investimentos em pesquisa por parte dos países mais ricos em países em condições menos favoráveis ${ }^{4}$.

Apesar de os recursos financeiros destinados à pesquisa e ao desenvolvimento em saúde no país ainda serem escassos ${ }^{6}$, na área odontológica notase um avanço em termos de produtividade a partir do século $\mathrm{XXI}^{7,8}$. Dados do Conselho Nacional de Desenvolvimento Científico e Tecnológico $(\mathrm{CNPq})^{9}$, revelaram que no ano 2000 a área de Ciências da Saúde representou 5,7\% das pesquisas brasileiras. Nessa época, as linhas de pesquisa em Odontologia totalizavam 892, enquanto em 2010 houve um aumento expressivo para $2029^{9}$.

Jara-Tracchia et al. ${ }^{10}$, em estudo acerca da taxa de publicação - razão entre a quantidade de resumos constantes dos anais da International Association for Dental Research (IADR) e publicações subsequentes na forma de artigos completos - de pesquisas provenientes de países latino-americanos, constataram que o Brasil supera em quase oito vezes a Argentina, com proporção de uma publicação para cada três pesquisas apresentadas.

O incentivo ao desenvolvimento da pesquisa consta das Diretrizes Curriculares Nacionais para os cursos de Odontologia, destacado no rol de competências e habilidades requeridas para o exercício profissional, sob o seguinte inciso do Art. 5.': "participar de investigações científicas sobre doenças e saúde bucal e estar preparado para aplicar os resultados de pesquisas para os cuidados de saúde" $"$.

Como forma de divulgação do que se produz, estão os eventos científicos, que ajudam a compor o currículo dos pesquisadores, ampliam a visibilidade das instituições de ensino e servem como parâmetro para se verificar a aceitação entre pares $^{12}$. Na área odontológica merece menção a Reunião Anual da SBPqO. Criada em 1983, tratase da divisão brasileira da IADR (www.sbpqo.org.br). Nesse evento é oportunizada a apresentação de pesquisas de todas as regiões do país, que abrangem diversas especialidades. Estudos dedicaram-se à investigação do que é publicado nos suplementos dessa Reunião, com ênfase em Saúde Bucal Coletiva ${ }^{13}$, Ética e Bioética $^{14}$, metodologias ativas de ensino ${ }^{15}$, Odontopediatria $^{16}$, análise qualitativa do que é produzido $^{17}$ e até mesmo comparações geográficas $^{16,18}$.

Quanto à Endodontia, pouco se sabe acerca da produção científica dessa área nas reuniões da $\mathrm{SBPqO}$, fato que motivou a realização da presente pesquisa, mediante avaliação dos resumos encontrados nos suplementos publicados entre 2010 e 2018.

\section{METODOLOGIA}

Com desenho observacional retrospectivo, esta análise bibliométrica foi realizada por meio de acesso aos suplementos do periódico Brazilian Oral Research, nos quais são publicados os anais das Reuniões Anuais da SBPqO, disponíveis em http://www.sbpqo.org.br.

Dois pesquisadores treinados e calibrados (kappa $=0,89)$ fizeram a coleta dos dados. Inicialmente foram selecionados apenas os resumos das pesquisas referentes aos painéis das categorias iniciante, aspirante e efetivo dos anos 2010 a 2018, exceto 2012, pois nesse ano ocorreu o encontro da IADR. Desta forma, chegou-se a 
um universo de 22.310 resumos publicados. Em seguida, os títulos foram lidos para identificar os resumos com temática em Endodontia. Nos casos em que havia dúvida, um terceiro avaliador foi consultado para o desempate. No total, 2516 resumos foram lidos na íntegra. Foram excluídos resumos de revisão, uma vez que se optou por avaliar somente pesquisas primárias, estudos com dentes decíduos - que apesar do envolvimento endodôntico estavam mais alinhados à Odontopediatria -, e estudos com pinos intracanal, por estarem em consonância com as áreas de Prótese e/ou Materiais Dentários.

Os textos foram catalogados em planilhas do Excel $^{\circledR}$ de acordo com as categorias temáticas relacionadas aos termos encontrados nos Descritores em Ciências da Saúde (DeCS www.decs.bvs.br) conforme o quadro 1. Para classificação quanto às regiões geográficas brasileiras (Norte, Nordeste, Centro-Oeste, Sul e Sudeste) considerou-se a instituição de origem mencionada no resumo.

Quadro 1. Categorias temáticas utilizadas no estudo

Medicação sistêmica: anti-inflamatórios, anestésicos - dosagem, complicações, efeitos adversos, controle da dor - pré-operatória e pós-operatória.

Anatomia: métodos, tomografia, ultrassonografia, microtomografia, microscopia, ressonância magnética, diafanização, radiografia analógica, radiografia digital.

Biossegurança: métodos, esterilização, desinfecção.

Microbiologia.

Citologia/histologia/genética: selamento apical, reparo, angiogênese/revascularização, célulastronco, engenharia tecidual, polimorfismo.

Diagnóstico: comparação entre métodos de diagnóstico para alterações pulpares e periapicais, oximetria, radiografia analógica, radiografia digital, termografia, teste de sensibilidade pulpar.

Epidemiologia: estudos de prevalência e de incidência, levantamentos epidemiológicos.

Materiais endodônticos: avaliação de propriedades físico-químicas, medicação intracanal, soluções irrigadoras, cimentos endodônticos, restauradores temporários, materiais obturadores, materiais cirúrgicos, clareadores internos.

Técnicas endodônticas: técnicas de preparo, de odontometria, métodos, técnicas de irrigação, técnicas de obturação, técnicas cirúrgicas, técnicas de clareamento interno, retratamento, laserterapia, terapia fotodinâmica.

Instrumentos endodônticos: desempenho de limas e de aparelhos de modo geral.

Traumatismo: meios de conservação, tratamento.

Desfecho clínico: infecções, acidentes e complicações.

Por meio do software SPSS, versão 21.0, teste Qui-quadrado foi aplicado para a busca de procedeu-se à análise univariada a fim de se possíveis associações $(\alpha=5 \%)$ entre a região, o conhecer a distribuição dos dados. Em seguida, o ano e a categoria temática da pesquisa. 


\section{RESULTADOS}

Um total de 22.310 trabalhos foi obtido no período analisado. Destes, 2516 eram da área de Endodontia e foram selecionados para compor a amostra. Os mesmos foram identificados quanto ao período em análise, conforme a figura 1 .

Em 2010, 375 resumos identificados nos suplementos eram da área de Endodontia, totalizando $13,4 \%$ no ano, o maior percentual no período analisado (tabela 1).

Quanto às categorias temáticas abordadas, constatou-se maior ênfase à análise das propriedades de materiais $(27,1 \%)$, pesquisas de técnicas $(20,6 \%)$ e de instrumentos $(13,0 \%)$. Pouco expressivos foram os percentuais de estudos sobre desfecho clínico (1,0\%), biossegurança $(1,2 \%)$ e terapêutica $(1,7 \%)$ (tabela 2).

$\mathrm{Na}$ avaliação da distribuição nacional dos resumos avaliados, a região do país com maiores taxas de concentração de pesquisas foi a Sudeste $(65,5 \%)$, seguida pelas regiões Sul $(17,2 \%)$ e Centro-Oeste $(6,8 \%)$ (tabela 3$)$.

$\mathrm{Na}$ análise de associação, foi encontrada diferença significativa entre região e ano $(\mathrm{p}=$ 0,006), porém não entre região e categoria temática $(\mathrm{p}=0,149)$. A participação da região Centro-Oeste no ano de 2010 acabou diferindo das demais, exceto da Nordeste. Já em 2015 esta região apresentou valores diferentes da região Sudeste. Nos demais anos esse comportamento não ocorreu (tabela 4).

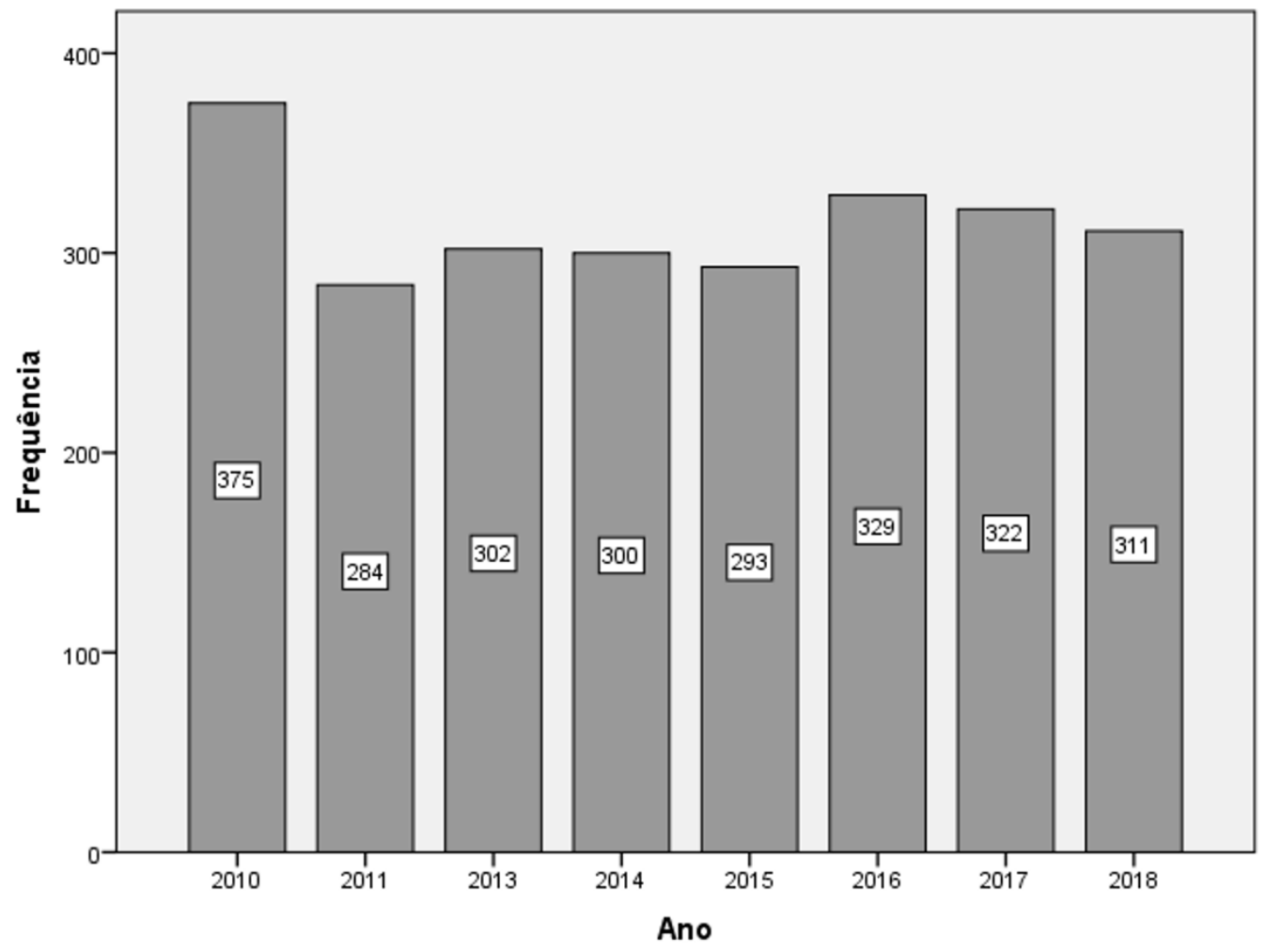

Figura 1. Distribuição dos resumos de Endodontia publicados nos suplementos das reuniões da SBPqO, de 2010 a $2018(n=2516)$ 
Tabela 1. Distribuição de resumos identificados nos suplementos das reuniões da SBPqO, que preencheram os critérios de inclusão na amostra, de 2010 a 2018

\begin{tabular}{lcc}
\hline Ano & Total & $\begin{array}{c}\text { Endodontia } \\
\mathbf{n}(\boldsymbol{\%})\end{array}$ \\
\hline 2010 & 2801 & $375(13,4)$ \\
2011 & 2546 & $284(11,1)$ \\
2013 & 2505 & $302(12,1)$ \\
2014 & 2490 & $300(12,1)$ \\
2015 & 2598 & $293(11,3)$ \\
2016 & 3044 & $329(10,8)$ \\
2017 & 3125 & $322(12,8)$ \\
2018 & 3201 & $311(12,4)$ \\
\hline
\end{tabular}

Tabela 2. Distribuição das categorias temáticas dos resumos de Endodontia publicados nos anais das Reuniões da SBPqO, de 2010 a 2018

\begin{tabular}{lc}
\hline Categoria temática & $\mathbf{n}(\mathbf{\%})$ \\
\hline Terapêutica & $43(1,7)$ \\
Anatomia & $154(6,1)$ \\
Biossegurança & $30(1,2)$ \\
Microbiologia & $236(9,4)$ \\
Citologia/Histologia/Genética & $261(10,4)$ \\
Diagnóstico & $120(4,8)$ \\
Epidemiologia & $62(2,5)$ \\
Materiais & $682(27,1)$ \\
Técnicas & $518(20,6)$ \\
Instrumentos & $328(13,0)$ \\
Traumatismo & $57(2,3)$ \\
Desfecho clínico & $25(1,0)$ \\
\hline Total & $\mathbf{2 5 1 6 ( 1 0 0 , 0 )}$ \\
\hline
\end{tabular}

Tabela 3. Distribuição dos resumos de Endodontia publicados nos anais das Reuniões da SBPqO em relação à região do país, de 2010 a 2018

\begin{tabular}{lc}
\hline Região & $\mathbf{n}(\boldsymbol{\%})$ \\
\hline Sudeste & $1648(65,5)$ \\
Sul & $433(17,2)$ \\
Centro-Oeste & $170(6,8)$ \\
Nordeste & $201(8,0)$ \\
Norte & $64(2,5)$ \\
\hline Total & $\mathbf{2 5 1 6 ( 1 0 0 , 0 )}$ \\
\hline
\end{tabular}


Tabela 4. Resultados da associação entre ano e região dos resumos publicados nos anais das Reuniões da SBPqO, de 2010 a 2018

\begin{tabular}{lcccccc}
\hline \multirow{2}{*}{ Ano } & \multicolumn{5}{c}{ Região } & \multicolumn{2}{c}{ Total } \\
\cline { 2 - 5 } & Sudeste & Sul & Centro-Oeste & Nordeste & Norte & \\
\hline 2010 & $263 \mathrm{a}$ & $61 \mathrm{a}$ & $10 \mathrm{~b}$ & $37 \mathrm{a}$ & $4 \mathrm{a}, \mathrm{b}$ & 375 \\
& $16,0 \%$ & $14,1 \%$ & $5,9 \%$ & $18,4 \%$ & $6,3 \%$ & $14,9 \%$ \\
2011 & $188 \mathrm{a}$ & $51 \mathrm{a}$ & $16 \mathrm{a}$ & $21 \mathrm{a}$ & $8 \mathrm{a}$ & 284 \\
& $11,4 \%$ & $11,8 \%$ & $9,4 \%$ & $10,4 \%$ & $12,5 \%$ & $11,3 \%$ \\
2013 & $189 \mathrm{a}$ & $58 \mathrm{a}$ & $31 \mathrm{a}$ & $18 \mathrm{a}$ & $6 \mathrm{a}$ & 302 \\
& $11,5 \%$ & $13,4 \%$ & $18,2 \%$ & $9,0 \%$ & $9,4 \%$ & $12,0 \%$ \\
2014 & $194 \mathrm{a}$ & $52 \mathrm{a}$ & $26 \mathrm{a}$ & $21 \mathrm{a}$ & $7 \mathrm{a}$ & 300 \\
& $11,8 \%$ & $12,0 \%$ & $15,3 \%$ & $10,4 \%$ & $10,9 \%$ & $11,9 \%$ \\
2015 & $179 \mathrm{a}$ & $53 \mathrm{a}, \mathrm{b}$ & $32 \mathrm{~b}$ & $21 \mathrm{a}, \mathrm{b}$ & $8 \mathrm{a}, \mathrm{b}$ & 293 \\
& $10,9 \%$ & $12,2 \%$ & $18,8 \%$ & $10,4 \%$ & $12,5 \%$ & $11,6 \%$ \\
2016 & $207 \mathrm{a}$ & $64 \mathrm{a}$ & $16 \mathrm{a}$ & $28 \mathrm{a}$ & $14 \mathrm{a}$ & 329 \\
& $12,6 \%$ & $14,8 \%$ & $9,4 \%$ & $13,9 \%$ & $21,9 \%$ & $13,1 \%$ \\
2017 & $214 \mathrm{a}$ & $45 \mathrm{a}$ & $19 \mathrm{a}$ & $35 \mathrm{a}$ & $9 \mathrm{a}$ & 322 \\
& $13,0 \%$ & $10,4 \%$ & $11,2 \%$ & $17,4 \%$ & $14,1 \%$ & $12,8 \%$ \\
2018 & $214 \mathrm{a}$ & $49 \mathrm{a}$ & $20 \mathrm{a}$ & $20 \mathrm{a}$ & $8 \mathrm{a}$ & 311 \\
& $13,0 \%$ & $11,3 \%$ & $11,8 \%$ & $10,0 \%$ & $12,5 \%$ & $12,4 \%$ \\
\hline
\end{tabular}

Nota: Letras diferentes indicam diferença estatisticamente significativa. Teste do Qui-quadrado $(\mathrm{p}<0,05)$

\section{DISCUSSÃO}

A pesquisa em saúde é fundamental para o desenvolvimento de um país ${ }^{1,2}$. No Brasil, o provimento de recursos destinados a essa finalidade é discreto $^{6}$, o que o distancia de patamares mais elevados atingidos por diversos países que tem altos índices de citação que os qualificam $^{19}$. Especificamente na Odontologia, a partir do século XXI houve um aumento da produtividade científica brasileira ${ }^{7-9}$, o que incentivou a realização de diversas avaliações em nível nacional ${ }^{13,17,18-23}$.

Dentre as formas de se oportunizar a divulgação do que se produz, estão os eventos científicos $^{12}$. Na área odontológica, destaca-se a Reunião Anual da SBPqO, dada a sua importância e representatividade enquanto entidade que difunde amplamente as pesquisas desenvolvidas. Uma série de estudos avaliaram o perfil de publicação brasileira em outras àreas, com o mesmo método bibliométrico empregado nesta pesquisa, a partir de dados disponibilizados pela $\mathrm{SBPqO}^{13-16}$. Outros avaliaram as áreas de conhecimento do $\mathrm{CNPq}$, região e estado do país, instituição, tipo de estudo e recebimento de fomento ${ }^{18,24}$, financiamento e categorias de análise (estudos biológicos, materiais, técnicos e/ ou sociais) $)^{17}$.

Uma das áreas com participação significativa na referida Reunião é a Endodontia. Durante o período avaliado foi identificado um equilíbrio em termos de quantidade de resumos publicados nos suplementos consultados. Dias et al. ${ }^{13}$ também relataram percentual significativo de ocorrência de trabalhos dessa área do período de 2001 a 2006.

A Endodontia é fortemente fundamentada em aspectos clínicos e dependente, de certo modo, de investigações acerca de materiais e de técnicas, fato comprovado pelos resultados da presente pesquisa, em que, quando analisadas as categorias temáticas, maior volume de estudos envolvia uso 
de materiais, técnicas e instrumentos. Na presente pesquisa foram incluídos apenas estudos primários em Endodontia, fato que possivelmente influenciou na alta concentração de desenhos com ênfase em técnicas, assim como observado em estudos anteriores ${ }^{13,17,18,24}$.

Apesar de a presente pesquisa ter sido destinada exclusivamente à área de Endodontia, constatou-se maior concentração de resumos publicados oriundos das regiões Sudeste e Sul do país. Esses achados corroboram estudos com propósito similar, de análise de dados da $\mathrm{SBPqO}$, cujos valores para a primeira região citada chegam a $70,1 \%{ }^{16}$ e $76,4 \%{ }^{18}$. Essa distribuição desigual da produção científica no Brasil indica que as regiões mais desenvolvidas socioeconomicamente comportam melhores indicadores de produção ${ }^{18}$. Nota-se que isso ocorre de modo paralelo ao maior desenvolvimento e quantidade de polos educacionais nessas mesmas regiões, além da maior concentração de programas de pósgraduação, o que impacta no aumento da produtividade $^{19}$. Valores indicados pelos censos do CNPq também corroboram esta distribuição ${ }^{22}$.

No cenário internacional o mesmo se repete, visto que o ranking produzido pelo Centro de Estudos em Ciência e Tecnologia (Centre for Science and Technology Studies - CWTS), da Universidade de Leiden, na Holanda, apontou em uma classificação de 2006 até 2019 que as cinco melhores instituições de ensino do Brasil são públicas, sendo que destas quatro estão da região Sudeste e uma na região Sul. Esta classificação considera a pesquisa acadêmica produzida pelas instituições com base nas publicações na base de dados Web of Science (https://www. leidenranking.com/ranking/2019/list).

Há que se ressaltar que o sucesso científicoacadêmico brasileiro deve estar pautado em uma agenda de pesquisas ${ }^{25}$, assim como organizações internacionais propõem ${ }^{1}$, desde que suportada principalmente pelo incentivo governamental ${ }^{7}$.
Muitas das fontes deste tipo de financiamento para as pesquisas odontológicas apresentadas na $\mathrm{SBPqO}$ provêm de órgãos públicos, como o $\mathrm{CNPq}^{18,24}$. Internacionalmente, o declínio de investimentos nas chamadas instituições odontológicas poderá afetar negativamente as pesquisas ${ }^{26}$, portanto atenção especial deve ser dada a reduções e cortes em orçamentos públicos destinados à Ciência e Tecnologia. O financiamento da pesquisa é considerado fundamental para a preservação da profissão odontológica ${ }^{27}$.

Os resultados aqui descritos permitiram retratar o perfil da pesquisa científica recente em Endodontia em um relevante evento científico nacional, o que de certa forma reflete o período de grande avanço tecnológico pelo qual passou a especialidade nos últimos tempos.

Por fim, como limitações do presente estudo, pode-se considerar a não extração de dados relativos a fontes de financiamento das pesquisas e classificação das instituições de ensino em públicas ou privadas, o que poderia ter permitido a expansão de outras comparações, que podem ser objeto de futuros estudos.

\section{CONCLUSÕES}

Com base na presente análise bibliométrica pode-se afirmar que a pesquisa em Endodontia tem ênfase em materiais e técnicas. No período avaliado, a maioria dos estudos apresentados nessa área foram desenvolvidos nas regiões Sudeste e Sul.

\section{AGRADECIMENTOS}

À Profa. Dra. Marili Doro de Andrade Deonízio, pelo auxílio no delineamento da metodologia deste projeto.
ABSTRACT
Scientific production in Endodontics at the Meeting of the Brazilian Society for Dental Research: bibliometric analysis from 2010 to 2018 
The annual meeting of the Brazilian Society of Dental Research (Sociedade Brasileira de Pesquisa Odontológica - SBPqO) is an important event of dental scientific production in the country. The evaluation of the profile of the presented research can identify characteristics and the evolution of Endodontics research. Through retrospective bibliometric analysis, meeting supplements from 2010 to 2018 were investigated to determine the scientific production in the area of Endodontics in SBPqO. The supplements were accessed online, via the institution's website, and the abstracts were analyzed according to region of origin: North, Northeast, Midwest, South and Southeast; and thematic categories: Therapeutics, Anatomy, Biosafety, Microbiology, Cytology/Genetics, Diagnosis, Epidemiology, Materials, Techniques, Instruments, Trauma and Clinical Outcome. Of the 22,310 abstracts published in the period, 2516 were selected from the Endodontics area. In 2010 there was the largest number of published abstracts ( $\mathrm{n}=$ 375). Prevailed the research focused on the analysis of materials $(27.1 \%)$ and techniques $(20.6 \%)$. The Southeast and South regions presented summary frequencies with values of $65.5 \%$ and $17.2 \%$, respectively, while the lowest participation was from the North region $(2.5 \%)$. The frequency of Endodontic research published in SBPqO supplement remained balanced over the analyzed period, with emphasis on objectives focused on materials and techniques, and higher productivity in the Southeast and South.

Descriptors: Research. Dentistry. Endodontics.

\section{REFERÊNCIAS}

1. Williams DM. The research agenda on oral health inequalities: the IADRGOHIRA initiative. Med Princ Pract. 2014; 23 (Sup 1):52-9.

2. Brasil. Ministério da Saúde. Secretaria de Ciência, Tecnologia e Insumos Estratégicos. Departamento de Ciência e Tecnologia. Por que pesquisa em saúde? Ministério da Saúde, Secretaria de Ciência, Tecnologia e Insumos Estratégicos, Departamento de Ciência e Tecnologia. - Brasília: Ministério da Saúde; 2007. 20 p.

3. Sundram FX. Scientific publication is dominated by First World countries. Ann Acad Med Singapore. 1998; 27(2):147.

4. Global Forum on Research and Innovation for Health. Forum 2015 Report; 2016 [Acesso em 23 jul 2018]. Disponível em: http://www.cohred.org/wp-content/uploads/ 2011/05/FORUM-2015-Report.pdf

5. Paraje G, Sadana R, Karam G. Increasing international gaps in health-related publications. Science. 2005; 308(5724):95960.

6. Silva RM, Caetano R. Um exame dos fluxos financeiros do Ministério da Saúde em pesquisa e desenvolvimento (2003-2005), segundo a Agenda Nacional de Prioridades de Pesquisa em Saúde. Cad Saúde Pública. 2011; 27(4):687-700.

7. Guimarães JA. A pesquisa médica e biomédica no Brasil: comparações com o desempenho científico brasileiro e mundial. Ciênc Saúde Coletiva. 2004; 9(2):303-27.

8. Pizzani L, Silva RC, Hayashi MCPI. Bases de dados e bibliometria: a presença da Educação Especial na base Medline. RBBD. 2008; 4(1):68-85.

9. Conselho Nacional de Desenvolvimento Científico e Tecnológico (CNPq). Diretório dos Grupos de Pesquisa no Brasil. Censos 2000-2010. 2010. [Acesso em 23 jul 2018]. Disponível em: http://lattes.cnpq.br/web/ dgp/por-area

10. Jara-Tracchia L, Aromando RF, Itoiz ME. Publication: Presentation rate in the Latin American region of the International Association for Dental Research. Acta Odontol Latinoam. 2010; 23(2):150-2.

11. Brasil. Ministério da Saúde. Secretaria da Ciência, Tecnologia e Insumos Estratégicos. Departamento de Ciência e Tecnologia. Fluxos de recursos financeiros para a 
pesquisa e desenvolvimento em saúde no Brasil: 2000-2002; 2006.

12. Hayashi MCPI, Guimarães VAL. A comunicação da ciência em eventos científicos na visão de pesquisadores. Em Questão. 2016; 22(3):161-83.

13. Dias AA, Narvai PC, Rêgo DM. Tendências da produção científica em odontologia no Brasil. Rev Panam Salud Publica. 2008, Jul; 24(1):54-60.

14. Marin F, Rebllo M, Mello ALSF, Finkler M. Ética e Bioética como temas de pesquisa em Odontologia: uma análise bibliométrica dos trabalhos apresentados nas reuniões da SBPqO. Rev ABENO. 2016; 16(4):51-60.

15. Maciel MMSA, Silva KBN, Melo JGA, Soares DM. Metodologia ativa aplicada ao ensino odontológico: um panorama nacional a partir de um estudo bibliométrico. Arch Health Invest. 2019; 8(2):74-8.

16. Morosini IAC, Otto WB, Carneiro VL, Oliveira LLRV, Oliveira DC, Ferreira FM. Profile of Brazilian scientific research in pediatric dentistry based on the 26th Annual Meeting of the SBPqO. Rev Odonto Cienc. 2012; 27(2):132-6.

17. Cormack EF, Silva Filho CF. A pesquisa científica odontológica no Brasil. Rev Assoc Paul Cir Dent. 2000; 54(3):242.

18. Cavalcanti AL, Melo TRNB, Barroso KMA, Souza FEC, Maia AMA, Silva ALO. Perfil da pesquisa científica em odontologia realizada no Brasil. Pesq Bras Odontopediatria Clín Integr. 2004; 4(2):99-104.

19. Guimarães R, Lourenço R, Cosac S. A pesquisa em epidemiologia no Brasil. Rev Saúde Pública. 2001; 35(4):321-40.

20. de Aquino SN, Martelli DRB, Bonan PRF, Laranjeira AL, Júnior HM. Produção científica odontológica e relação com agências de financiamento de pesquisa. Arq Odontol. 2009; 45(3):142-6.
21. Leles CR, Rocha SS, Simões PA, Compagnoni MA. Taxa de publicação, na forma de artigos completos, de resumos apresentados em evento científico de pesquisa odontológica. Rev Odontol UNESP. 2006; 35(3):141-7.

22. Vieira Júnior JR, Martelli DRB, Bonan PRF, Silveira MF, Martelli HM. Pesquisa odontológica no estado de Minas GeraisBrasil: relação entre a FAPEMIG e a produtividade científica. Arq Odontol. 2008; 44(4):11-6.

23. Amorim KPC, Alves MSCF, Germano RM. A construção do conhecimento na odontologia: a produção científica em debate. Acta Cir Bras. 2005; 20(1):12-5.

24. Xavier AFC, Silva ALO, Cavalcanti AL. Análise da produção científica em Odontologia no nordeste brasileiro com base em um congresso odontológico. Arq Odontol. 2011; 47(3):127-34.

25. Gomes D, Agnoletto IG, Souza ML, Spiger V, Jakymiu JRG, Fuji EC, et al. A produção científica da Odontologia e a Agenda Nacional de Prioridades de Pesquisa em Saúde. Rev ABENO. 2017; 17(2):11-21.

26. Ferland CL, O'Hayre M, Knosp WM, Fox $\mathrm{CH}$, Horsford DJ. The NIH's funding to US dental institutions from 2005 to 2014. J Dent Res. 2017; 96(1):10-6.

27. Feinberg M, Garcia LT, Polverini PJ, Fox $\mathrm{CH}$, Valachovic RW. The vital role of research funding in preserving the oral health of the public and the dental profession. J Am Dent Assoc. 2015; 146(6):355-6.

\section{Correspondência para:}

Marilisa Carneiro Leão Gabardo

e-mail: marilisagabardo@gmail.com

Rua Prof. Pedro Viriato Parigot de Souza, 5300

81280-330 Curitiba/PR 